ARTICLE

https://doi.org/10.1038/s41467-020-18374-z

\title{
High-speed III-V nanowire photodetector monolithically integrated on $\mathrm{Si}$
}

Svenja Mauthe (1) 1, Yannick Baumgartner (1) 1, Marilyne Sousa1, Qian Ding (1) 2, Marta D. Rossell (1) 1,3, Andreas Schenk ${ }^{2}$, Lukas Czornomaz ${ }^{1} \&$ Kirsten E. Moselund (i) ${ }^{1 凶}$

Direct epitaxial growth of III-Vs on silicon for optical emitters and detectors is an elusive goal. Nanowires enable the local integration of high-quality III-V material, but advanced devices are hampered by their high-aspect ratio vertical geometry. Here, we demonstrate the in-plane monolithic integration of an InGaAs nanostructure $p$-i-n photodetector on Si. Using free space coupling, photodetectors demonstrate a spectral response from 1200-1700 nm. The $60 \mathrm{~nm}$ thin devices, with footprints as low as $\sim 0.06 \mu \mathrm{m}^{2}$, provide an ultra-low capacitance which is key for high-speed operation. We demonstrate high-speed optical data reception with a nanostructure photodetector at $32 \mathrm{~Gb} \mathrm{~s}^{-1}$, enabled by a $3 \mathrm{~dB}$ bandwidth exceeding $\sim 25 \mathrm{GHz}$. When operated as light emitting diode, the $p-i-n$ devices emit around $1600 \mathrm{~nm}$, paving the way for future fully integrated optical links.

\footnotetext{
${ }^{1}$ IBM Research Europe, Säumerstr. 4, 8803 Rüschlikon, Switzerland. ${ }^{2}$ Department of Information Technology and Electrical Engineering, Integrated Systems Laboratory, ETH Zurich, Gloriastr. 35, 8092 Zurich, Switzerland. ${ }^{3}$ Electron Microscopy Center, Empa, Swiss Federal Laboratories for Materials Science and Technology, Überland Str. 129, 8600 Dübendorf, Switzerland.『email: kmo@zurich.ibm.com
} 
$\mathrm{D}$ ata traffic and integration density in electronic integrated circuits has increased drastically in the past decade, resulting in an increase in power consumption of computing resources worldwide ${ }^{1-3}$. A large fraction of this power is used to transmit data along resistive electrical interconnects. It has long been the goal to integrate optical interconnects on the electronic chips itself, and recent progress in scaling and performance of integrated photonic components has made this vision more tangible. To present a viable alternative to copper interconnects, optical links need to provide significantly better energy efficiency, while supporting higher bandwidth densities and lower cost per bit. This requires physically scaled components with ultra-low capacitance that are densely integrated with their control electronics by leveraging monolithic integration technologies 4 . While some optical link schemes are based on off-chip lasers ${ }^{5,6}$, others foresee the integration of multiple lasers or light emitting diodes (LEDs) on chip ${ }^{7}$. In both cases, densely integrated highspeed photodetectors are key components for on-chip optical transceivers. State-of-the-art integrated photodetectors are commonly based on germanium, since this material is readily available in CMOS foundries to enhance hole mobility in compressively strained p-channel transistors. Scaled Ge photodetectors have reached a high level of maturity, with detection bandwidths exceeding $70 \mathrm{GHz}$ and operation at $100 \mathrm{GBd}^{8,9}$, however, they often tend to suffer from high dark currents and low absorption due to the indirect nature of Ge. III-V materials on the other hand, exhibit higher absorption coefficients and due to their direct tunable bandgap, they also enable active optical emitters such as lasers ${ }^{10-13}$ or LEDs ${ }^{14-16}$. The integration on $\mathrm{Si}$, however, is significantly more challenging because of the substantial lattice, polarity, and thermal mismatch. A seamless integration scheme between scaled photonic and electronic components will require a local monolithic integration approach. Nanowire (NW) techniques are of interest due to their small epitaxial interface with Si which allows for the growth of high-quality single crystalline material despite a high degree of lattice mismatch. Furthermore, the small size of NW detectors results in a capacitance in the sub-fF regime, which is required for both low-power and high-speed operation. Different fabrication techniques have been pursued from growing III-V NWs on III-V substrates ${ }^{17-19}$ to catalyzed growth on Si using Au particles ${ }^{20,21}$ or selective area III-V growth on $\mathrm{Si}^{15,22-24}$. Existing $\mathrm{NW}$ technologies however, either result in vertically oriented devices ${ }^{15,18,23}$ or when integrated laterally, i.e., lying on the substrate, are based on pickand-place techniques ${ }^{17,19,21}$. So far, neither of these approaches are suitable for large-scale integration or waveguide coupling, nor are they compatible with conventional CMOS process flows. Moreover, to the best of our knowledge, there has only been a single demonstration of NW detectors monolithically integrated on $\mathrm{Si}$ operating in the $\mathrm{GHz}$ regime. In ref. ${ }^{15}$, Chen et al. demonstrated an InGaAs core-shell $p-i-n$ structure with a $3 \mathrm{~dB}$ bandwidth of 3.1 $\mathrm{GHz}$, a factor of 10 lower than what is required for optical interconnects.

In this work, we demonstrate the first ultrathin and monolithically integrated nanostructure $p-i-n$ photodetector on $\mathrm{Si}$ with a responsivity reaching $\sim 0.68 \mathrm{~A} \mathrm{~W}^{-1}$ in the O-band (1260-1360 $\mathrm{nm})$. We demonstrate for the first time high-speed optical data reception at $32 \mathrm{~Gb} \mathrm{~s}^{-1}$ and a $3 \mathrm{~dB}$ bandwidth exceeding $\sim 25 \mathrm{GHz}$ using a nanostructure photodetector, as well as photodetection in the C-band $(1530-1565 \mathrm{~nm})$. Moreover, when operated as an LED, the fabricated $p-i-n$ devices show light emission in the Cband, thus paving the way for a fully integrated optical link.

\section{Results}

Integration of III-V photonic devices on $\mathrm{Si}$ using TASE. Figure 1 shows our vision of a future III-V based electro-optical

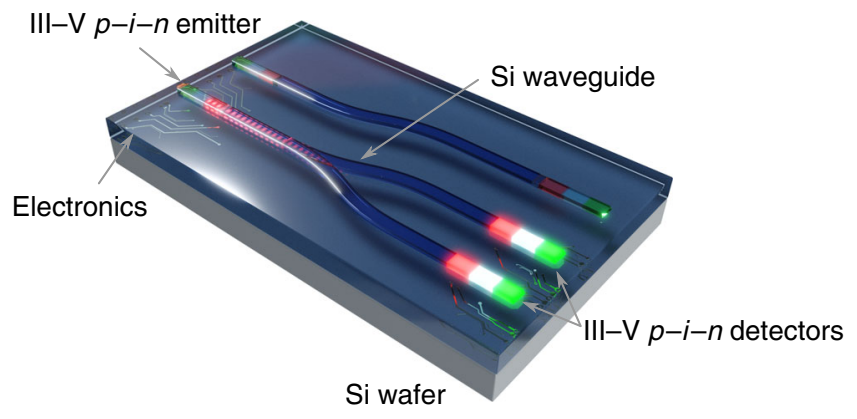

Fig. 1 Vision of a future all-optical link fabricated via TASE. Both emitter and detector can be integrated in-plane and butt-coupled to a Si waveguide. The present work employs free-space coupling but paves the way for future waveguide-coupled devices.

platform on Si with optical devices, such as photodetector and light source, directly coupled to a $\mathrm{Si}$ waveguide. To make this platform a reality, III-V materials have to be integrated directly on $\mathrm{Si}$, in-plane with silicon features and with lateral in-situ doping profiles to enable device operation. In this work, we propose a novel device integration scheme that enables in-plane junctions and direct growth of III-V material on Si waveguides. Devices are grown on $\mathrm{Si}$ and characterized using free-space optical coupling, as current dimensions of the silicon waveguide onto which the $p-i-n$ structure is grown are too small to support a propagating optical mode. A key feature of our devices is an inplane doping profile resulting in a horizontal $p-i-n$ nanostructure compared to commonly used vertical or radial $p-i-n$ stacks. Using template-assisted selective epitaxy (TASE ${ }^{25}$ ) combined with in-situ doping, we integrated InGaAs homojunctions epitaxially on Si. The in-plane approach may allow for easy waveguide coupling in the future by directly growing the III-V active material as an extension to SOI waveguides. This technique has previously been used to demonstrate in-plane electronic devices ${ }^{26}$ and optically pumped microdisks ${ }^{12}$. The concept of TASE relies on an oxide template to guide the epitaxial growth as illustrated in Fig. 2a. The top Si layer of an SOI wafer is patterned, covered with $\mathrm{SiO}_{2}$ forming an oxide template, and the Si partially etched back through an opening in the $\mathrm{SiO}_{2}$ to form a hollow template. III-V material is grown from the $\mathrm{Si}$ seed into the hollow $\mathrm{SiO}_{2}$ template. Structures can be densely integrated since the location of the III-V is defined lithographically in the same step as the silicon passives. Moreover, the technique offers co-integration of multiple III-Vs ${ }^{27}$, heterojunctions ${ }^{28}$, and the possibility of integrating quantum wells. The fabrication is performed on standard SOI substrates with $2 \mu \mathrm{m}$ buried oxide (BOX). More details on the fabrication can be found in the "Methods" section. In this work, nanostructure shaped $\mathrm{SiO}_{2}$ templates are formed following the fabrication steps depicted in Fig. 2a. Starting at the Si seed, InGaAs is grown into the hollow oxide template using metalorganic chemical vapor deposition (MOCVD) at $550^{\circ} \mathrm{C}$. In-situ $p$ - and $n$-doped regions are achieved by adding doping precursors during the growth and their extension controlled by the growth duration. Starting at the Si seed, $\sim 330 \mathrm{~nm} p$-doped InGaAs are grown, followed by $\sim 330 \mathrm{~nm}$ intrinsic InGaAs, and finally $\sim 330$ $\mathrm{nm} n$-doped InGaAs (the growth sequence is shown in Fig. $2 \mathrm{~b}$ and more detail is provided in the Supplementary Notes 1 and 2). The final $p-i-n$ InGaAs nanostructures exhibit a length of $\sim 1 \mu \mathrm{m}$, a height of $60 \mathrm{~nm}$, and widths between $60 \mathrm{~nm}$ and $500 \mathrm{~nm}$ as defined in the design. Electrical contacts are formed using a $\mathrm{Ni} /$ $\mathrm{Au}$ lift-off process. Figure $2 \mathrm{c}$ and $\mathrm{d}$ show an SEM image of an as grown and contacted device and a cross-section along the center of the nanostructure, respectively. An activation energy of $\sim 200 \mathrm{meV}$ is measured on the devices (see Supplementary Note 6) 
a

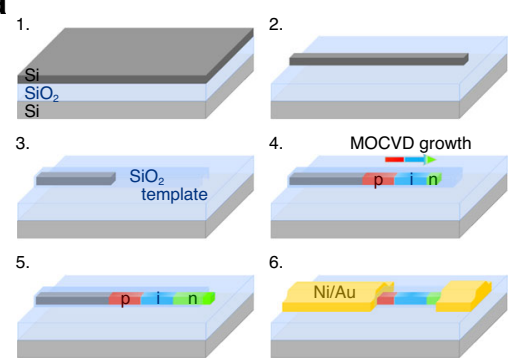

b

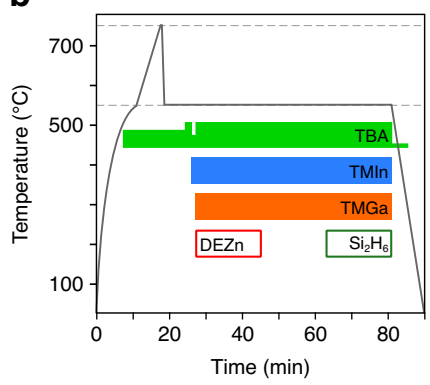

C

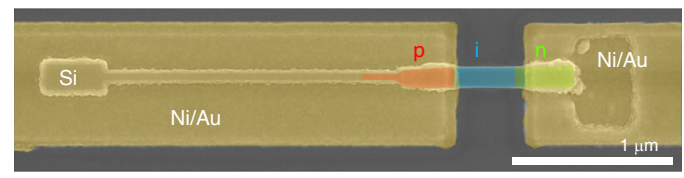

d

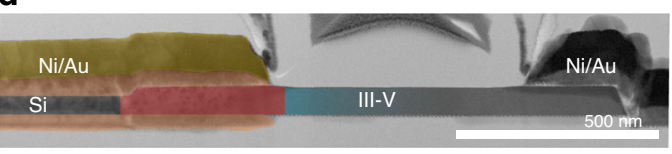

Fig. 2 Template-assisted selective epitaxy fabrication process. a Process steps during TASE. 1. SOI wafer. 2. Patterned top Si layer. 3. Partially hollow $\mathrm{SiO}_{2}$ template with $\mathrm{Si}$ seed. 4. MOCVD growth of $p-i-n \operatorname{InGaAs}$. The arrow indicates the growth direction. 5. $p-i-n$ nanostructure after growth. 6. Ni/Au contacts on nanostructure. $\mathbf{b}$ Schematic of the $p-i-n \operatorname{InGaAs}$ MOCVD growth sequence. The colored regions correspond to the turning on of the flow of precursors. First, an annealing step is performed to desorb water residues. Next, a short InAs seed is deposited for nucleation followed by the $p-i-n$ InGaAs growth. Details can be found in the Supplementary Note 1. c False-colored SEM top view of $p-i-n$ nanostructure. d Bright field STEM image of a crosssection of the nanostructure in (c). Colors indicate materials in the left part.

a

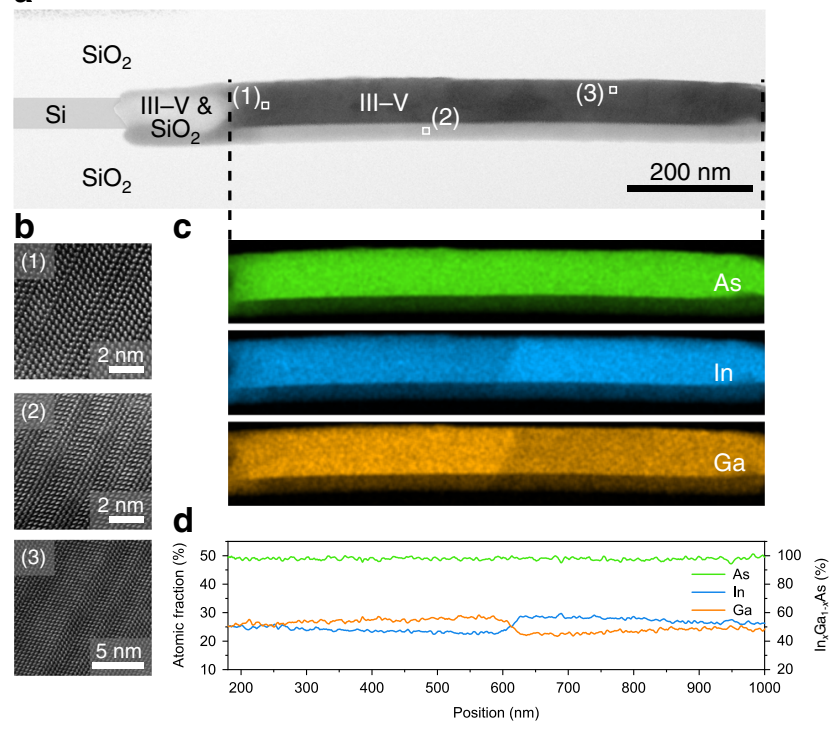

Fig. 3 Epitaxial single crystalline InGaAs nanostructure. a Bright field STEM overview image of a $200 \mathrm{~nm}$ wide $p-i-n$ nanostructure without contacts. The III-V material is grown from the $\mathrm{Si}$ in a funnel shape and hence, results in a thinner III- $\mathrm{V}$ region overlaid with $\mathrm{SiO}_{2}$ at the $\mathrm{Si}$ interface which is visible as a brighter region. The $\mathrm{Si}$ seed is false colored to enhance visibility. $\mathbf{b}$ High resolution annular dark field STEM images taken at marked positions in (a). c EDS analysis of the different elements in the $\ln _{x} \mathrm{Ga}_{1-x} A s$ nanostructure. d Extracted atomic fraction along the nanostructure.

Compositional line profiles obtained by averaging the integrated intensities over the nanostructure diameter.

suggesting a small energy barrier at the p-type contact. With resulting ultra-small footprints as low as $\sim 0.06 \mu \mathrm{m}^{2}$, the fabricated $p-i-n$ nanostructures are excellent candidates for densely integrated Si PICs. The scaled dimensions of the device suggest a junction capacitance below $0.1 \mathrm{fF}$.

Detailed material characterization. To investigate the material quality, scanning electron microscopy (SEM), scanning transmission electron microscopy (STEM), and energy dispersive Xray spectroscopy (EDS) analysis are performed on several focused ion beam prepared lamellas containing a cross-section of a nanostructure (see Fig. 3a). The STEM analysis reveals a single crystalline structure epitaxially aligned with the Si seed crystalline orientation with misfit dislocations locally confined to the Si/ III-V interface. High resolution images depicted in Fig. 3b demonstrate the absence of threading dislocations in this device but reveal stacking faults and short wurtzite (WZ) segments in the zincblende $(\mathrm{ZB})$ InGaAs structure. Figure $3 \mathrm{c}$ shows the EDS elemental maps of As, In, and $\mathrm{Ga}$ along the nanostructure, revealing an average $\operatorname{In}_{x} \mathrm{Ga}_{1-x}$ As concentration of $x \sim 50 \%$ (see Fig. 3d). Moreover, small variations in the In content between $x \sim 47 \%$ and $x \sim 53 \%$ are observed corresponding to unstrained theoretical ZB bulk bandgaps of $E_{\mathrm{G}}\left(\operatorname{In}_{0.47} \mathrm{Ga}_{0.53} \mathrm{As}\right) \sim 0.78 \mathrm{eV}$ and $E_{\mathrm{G}}\left(\mathrm{In}_{0.53} \mathrm{Ga}_{0.47} \mathrm{As}\right) \sim 0.74 \mathrm{eV}$, respectively. We believe that the composition variation can be correlated with the switching of doping precursors during MOCVD growth. This is consistent with previous work where similar effects were observed ${ }^{29,30}$.

In-plane nanostructure photodetectors. Photodetectors are characterized in different optical set ups to evaluate their spectral response and high-speed operation. Extensive numerical simulation using "Synopsys Sentaurus" 31 is used to analyze their behavior. First, the dark current performance of the fabricated photodetectors is characterized without optical illumination (dark). A low dark current is desired, enabling a high signal-tonoise ratio. The $p-i-n$ nanostructures show diode-like $I-V$ characteristics as depicted in Fig. 4a. Devices of $60 \mathrm{~nm}$ width exhibit dark currents in reverse bias as low as $1.7 \mathrm{nA}$ at $-2 \mathrm{~V}$, for devices with $200 \mathrm{~nm}$ and $500 \mathrm{~nm}$ width dark currents of $15 \mathrm{nA}$ and $36 \mathrm{nA}$ at $-2 \mathrm{~V}$ were measured, respectively (more details in Supplementary Note 5). These values compare well with dark currents measured in bonded, high material quality III-V photodetectors $^{32}$ as well as in conventional InGaAs photodetectors ${ }^{33}$ and are lower than values achieved in grown InGaAs metal-semiconductor-metal NW photodetectors ${ }^{20}$. To investigate the spectral response of the photodetectors, devices are illuminated with a ps-pulsed supercontinuum laser $(78 \mathrm{MHz}$ repetition rate) and their $I-V$ characteristics measured. Figure $4 \mathrm{~b}$ depicts $I-V$ curves of a single $200 \mathrm{~nm}$ wide detector with increasing incident ps-pulsed optical power at $1346 \mathrm{~nm}$. Next, the incident laser wavelength is varied between 1000 and $1750 \mathrm{~nm}$. Figure $4 \mathrm{c}$ compares the current density for different device widths normalized to their respective maximum. The spectral responses of the devices are similar with light absorption between 1200 and $1700 \mathrm{~nm}$ and a maximum around $1350 \mathrm{~nm}$. A flat spectral response as for bulk InGaAs is not expected due to the combination of the small device thickness and the free-space coupling, 
a

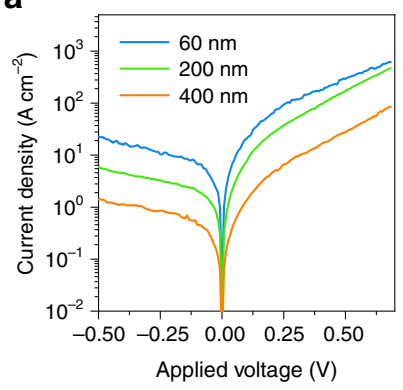

d

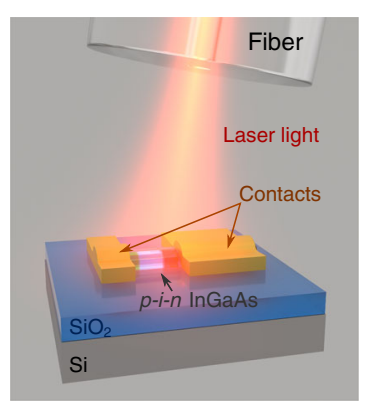

b

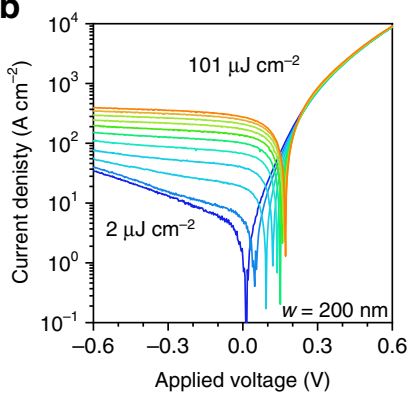

e

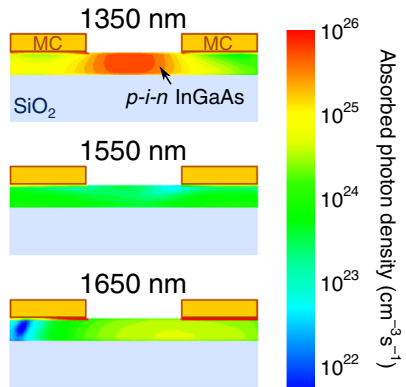

C

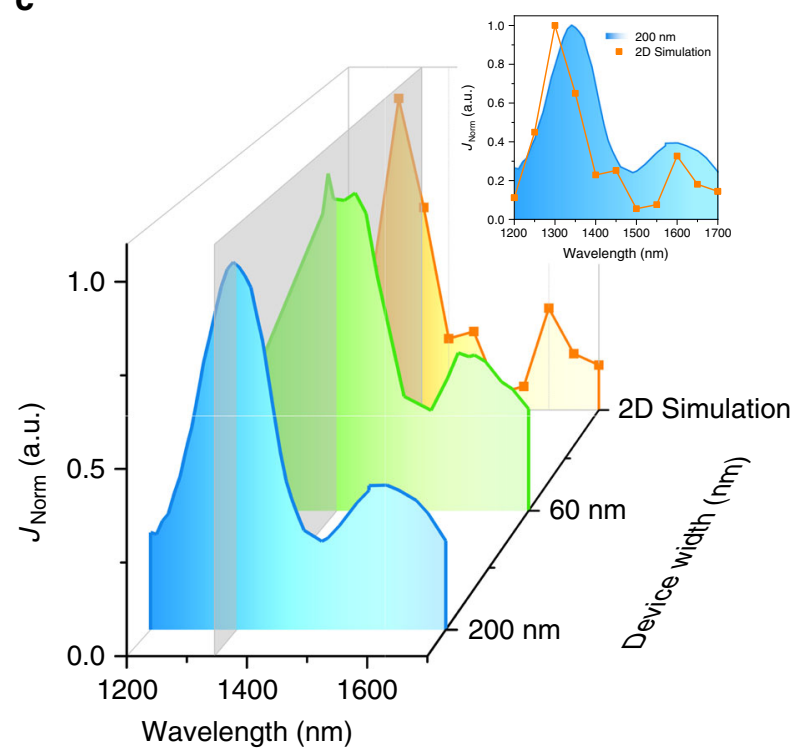

Fig. 4 Static electro-optical characterization of the $\boldsymbol{p}$-i-n InGaAs photodetectors. a $I-V$ curves for devices with different widths. $\mathbf{b} I-V$ curves for increasing incident laser powers $(1346 \mathrm{~nm})$ of the $200 \mathrm{~nm}$ wide device. c Normalized spectral response for devices with different widths at $-0.5 \mathrm{~V}$ bias as well as for a simulated device (2D). The current density is normalized for better visibility, however the current density for individual devices is in the same order of magnitude of $100 \mathrm{~A} \mathrm{~cm}^{-2}$. The inset depicts a comparison of the experimentally obtained spectral response for a $200 \mathrm{~nm}$ wide device and the simulated 2D device using electro-optical simulations. d Schematic of the free-space coupling for electro-optical devices. e Simulated absorbed photon density for different incident wavelengths (1350, 1550, and $1650 \mathrm{~nm}$ ) on $p-i-n \operatorname{lnGaAs}$ devices with metal contacts (MC). The devices are $60 \mathrm{~nm}$ high and $1 \mu \mathrm{m}$ long. The $z$-direction is enlarged by a factor of 2 for better visibility.

which favors the absorption of shorter wavelengths closer to the surface. To further understand the spectral response of the devices we perform 2D electro-optical simulations using "Synopsys Sentaurus" simulation software. A device structure as depicted in Fig. 4d is simulated with monochromatic light impinging under $10^{\circ}$ corresponding to the free-space light coupling in the experimental setup. The simulations reveal multiple reflections of the incident light between the $\mathrm{Si}$ substrate, the $\mathrm{SiO}_{2}$ BOX layer, and within the device between its contacts (see Supplementary Note 9). This results in an incident wavelength dependent optical generation in the structure as depicted in Fig. 4e and hence, a spectral response with two distinct absorption peaks around 1350 and $1650 \mathrm{~nm}$ (Fig. 4c). This simulated spectral response confirms the experimentally measured data.

An important measure to characterize a photodetector is its efficiency to convert incident photons to electron-hole pairs, namely the responsivity. Whereas, the responsivity is easily measured in a fully integrated platform, in the case of NWs or nanostructures it can be calculated based on the measured photocurrent and an estimation of the incident illumination power ${ }^{21,24}$. To determine the responsivity at the maximum absorption, we excite the $p-i-n$ structures with a continuous wave (cw) laser at $1346 \mathrm{~nm}$. The incident light intensity is calculated taking the position of the optical fiber and the illuminated device area into account (see details in Supplementary Note 10 along with the bias-dependent responsivity). The measured device shows a responsivity up to $0.68 \mathrm{~A} \mathrm{~W}^{-1}$ at $-2 \mathrm{~V}$ and a quantum efficiency up to $62 \%$. If we assume that carriers generated in the doped regions also contribute to the photocurrent, the calculated responsivity drops to $\sim 0.2 \mathrm{~A} \mathrm{~W}^{-1}$. These values are very promising and compare well with values achieved in similar InGaAs structures ${ }^{15}$. A detailed analysis of the responsivity can be found in the Supplementary Note 10.
Electrically and optically pumped light emission. To further investigate the optical spectrum of the $p-i-n$ structures, photoluminescence (PL) spectroscopy is performed and the obtained spectrum depicted in Fig. 5a. The spectrum reveals an emission peak around $\sim 1600 \mathrm{~nm}$, corresponding to expected values from bulk $\mathrm{ZB} \mathrm{In} \mathrm{Ga}_{1-x} \mathrm{As}$ for the determined compositions in Fig. $3 \mathrm{~d}$. Moreover, the obtained PL spectrum agrees well with the spectral response of the nanostructure, which is influenced by reflections at the various interfaces. Furthermore, it may be influenced by the relatively high pump powers that are required to get sufficient light output from the nanostructures ${ }^{34,35}$.

Electroluminescence (EL) with a single emission peak in the desired $\mathrm{C}$ telecommunication band is observed when devices are operated as an LED in forward bias, as shown in Fig. 5b. With increasing electrical pumping, the intensity of the EL increases. The EL spectrum matches well the second emission peak measured in PL. These initial measurements demonstrate the dual functionality of the $p-i-n$ nanostructures for both light detection and emission.

High-speed operation. High-speed operation is key for future applications and one of the main motivations for moving from electronics to optical signal propagation. We measure the frequency response of the photodetectors and determine their $3 \mathrm{~dB}$ bandwidth using a modulated optical signal at $1346 \mathrm{~nm}$ illuminated from the top. Figure 6a depicts the measured frequency response (scattering parameter $S_{21}$ ) of a $200 \mathrm{~nm}$ wide photodetector biased at $-1.5 \mathrm{~V}$. The plot reveals a $3 \mathrm{~dB}$ bandwidth beyond our measurement capabilities $(\sim 25-30 \mathrm{GHz})$. The demonstrated $25 \mathrm{GHz}$ bandwidth is, to the best of our knowledge, the highest reported value for nanostructure photodetectors. To further evaluate the performance at high baud rates, we acquire 

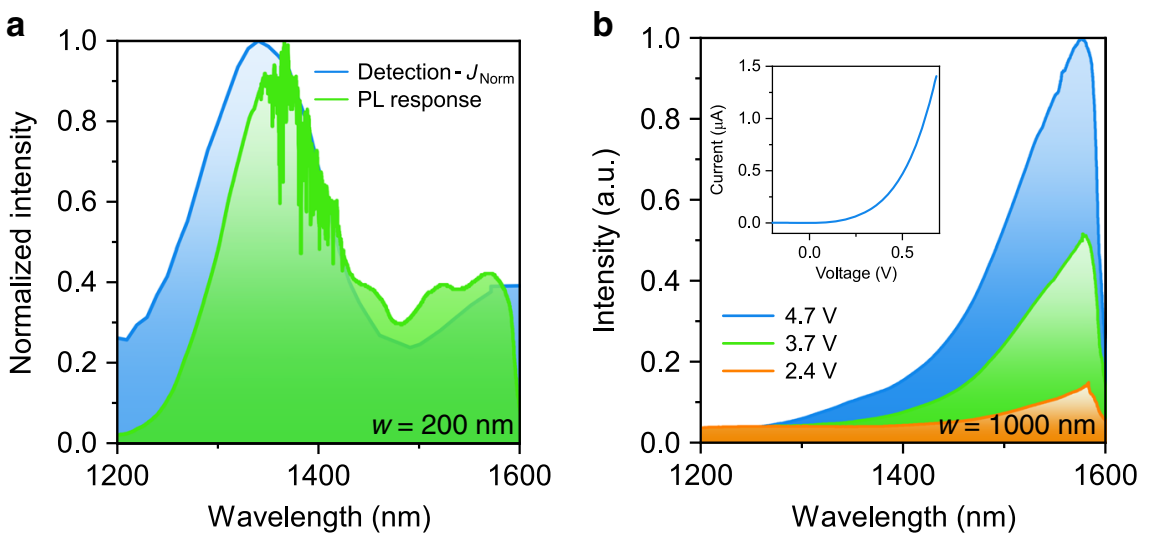

Fig. 5 Electrically and optically pumped light emission. a Measured PL and spectral absorption response spectra $(w=200 \mathrm{~nm})$. b EL from a $\sim \mu \mathrm{m}$ wide device for different applied voltages. Close to $1600 \mathrm{~nm}$, the cutoff of the measurement detector connected to the spectrometer is visible. The inset shows the $I-V$ curve in forward bias.

a

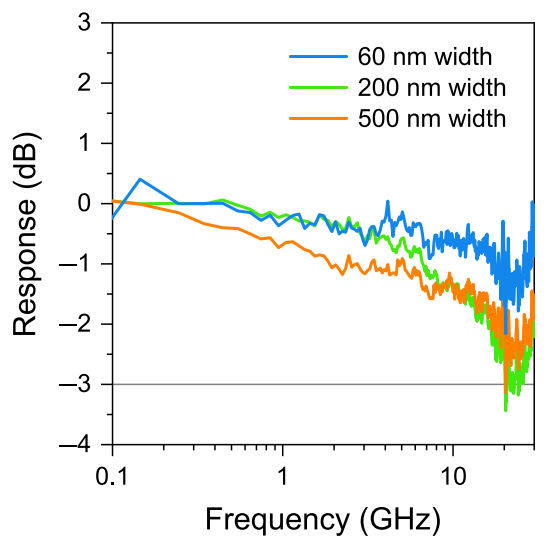

b

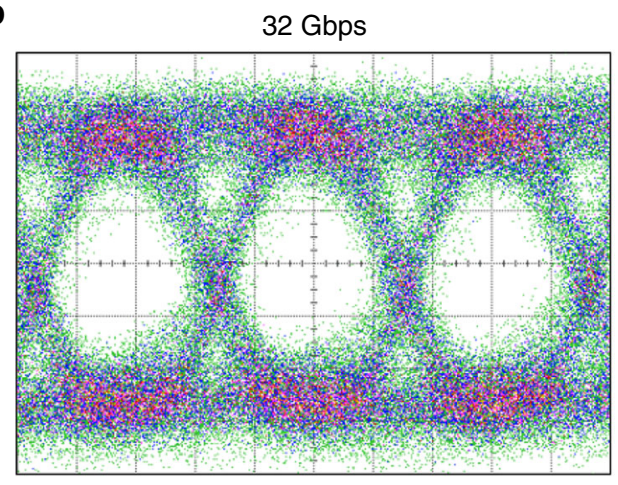

Fig. 6 High-speed data reception. a $S_{21}$ measurements for devices with different widths. The line at $-3 \mathrm{~dB}$ marks the $3 \mathrm{~dB}$ limit. $\mathbf{b} 32 \mathrm{~Gb} \mathrm{~s}^{-1}$ eye diagram measured on a $500 \mathrm{~nm}$ wide device at $-2 \mathrm{~V}$ applied bias ( $1.5 \mathrm{mV} \mathrm{div}^{-1}, 10 \mathrm{ps}^{-1} \mathrm{div}^{-1}$ ). Averaging was set to 128.

electrical eye-diagrams. A non-return-to-zero on-off Keying pseudo random binary sequence of $2^{7}-1$ bits is sent onto the photodetectors and their electrical response measured on a sampling scope. Figure $6 \mathrm{~b}$ depicts an eye diagram obtained at $32 \mathrm{~Gb} \mathrm{~s}^{-1}$. A clear eye opening at high data rates is visible, confirming the high-speed nature of our photodetectors.

\section{Discussion}

In conclusion, the lateral integration enabled by TASE permits the in-plane integration of homo- and heterojunctions within a nanostructure geometry. The seamless integration with silicon along with the in-plane profiles, which are more easily compatible with conventional processing, are essential for future VLSI electronic-photonic circuits on-chip. The monolithically integrated InGaAs $p-i-n$ photodetectors can cover several telecommunication bands, with a maximum responsivity up to 0.68 $\mathrm{A} \mathrm{W}^{-1}$ at $1346 \mathrm{~nm}$. Furthermore, detection bandwidths exceeding $25 \mathrm{GHz}$ were demonstrated, enabling high-speed operation at $32 \mathrm{GBd}$. TCAD simulations are used to analyze device behavior, the simulated spectral response matches the experimental one, with two peaks at around 1350 and $1600 \mathrm{~nm}$. We believe that in a future waveguide-coupled approach with device diameters supporting a single mode, the performance will be comparable or better due to a strong light confinement in the waveguide structure and the potential for implementing a longer absorption region.
When operated as an LED, EL in the C-band is observed. The presented work highlights the great potential for dense integration of III-V electro-optical devices on silicon, which paves the way for the future integration with Si CMOS electronics, and hence will fuel applications such as energy efficient and highspeed optical interconnects for intra-chip or chip-to-chip communication, advanced image sensing, laser radar (LiDAR), or integrated neurophotonics. Since this technique relies on the direct growth extended in-plane from $\mathrm{Si}$ features, future devices can be grown directly from a Si waveguide enabling a full optical link with direct coupling.

\section{Methods}

Sample preparation. In a first step, the top Si layer of an SOI wafer is thinned down to $60 \mathrm{~nm}$ defining the height of the final structures. Next, dimensions, shape, and location of the final structures are lithographically defined and patterned using e-beam lithography and an inductively coupled plasma dry etch of the top silicon layer. The patterned Si structures are partially replaced by III-V material in the final step. This one-step lithographic process allows for a precise alignment of III-V and silicon features. In total, $50 \mathrm{~nm}$ atomic layer deposition $\mathrm{SiO}_{2}$ and $150 \mathrm{~nm}$ $\mathrm{SiO}_{2}$ using plasma enhanced chemical vapor deposition are deposited covering the patterned Si structures and serving as an oxide template for the final III-V growth. Openings are locally etched into the $\mathrm{SiO}_{2}$ layer partially exposing the underlaying patterned $\mathrm{Si}$ structures at one end. In a subsequent tetramethylammonium

hydroxide wet etch, the patterned $\mathrm{Si}$ is partially etched resulting in a hollow oxide template with a remaining $\mathrm{Si}$ seed at one extremity. The wet etch process allows for a precise control of the etch-back distance and results in a smooth surface at the $\mathrm{Si}$ where the growth will nucleate. Finally, the hollow $\mathrm{SiO}_{2}$ cavity is filled with epitaxial III-V material using MOCVD growth at $550^{\circ} \mathrm{C}$ : a small $\sim 20 \mathrm{~nm}$ long region 
of indium arsenide (InAs, precursors: TMIn, TBA) is grown which selectively nucleates at the $\mathrm{Si}$ interface. Next, InGaAs is grown using TMIn, TBA, and TBGa precursors with a V/III ratio of 0.27 . More details can be found in Supplementary Note 1. By adding dopants, the III-V material can be in situ $p$ - or $n$-doped. In this work, DEZn and $\mathrm{Si}_{2} \mathrm{H}_{6}$ are added as $p$ - and $n$-dopants, respectively. Details on the doping concentration can be found in the Supplementary Note 2. Contacts were formed using a conventional $\mathrm{Ni} / \mathrm{Au}$ lift-off process.

Structural characterization. To analyze the structural quality of the devices, crosssectional lamellas from five different devices were investigated. The lamellas were prepared using a FEI Helios Nanolab $450 \mathrm{~S}$ focused ion beam. The cut was performed along the growth direction to reveal the $\mathrm{Si}$ seed and its nucleation interface with the III-V material. The lamellas were further investigated by STEM using two different instruments. High resolution images were generated using a double spherical aberration-corrected JEOL JEM-ARM200F microscope operated at $200 \mathrm{kV}$, while the EDS spectrum image was acquired with a FEI Titan Themis operated at $300 \mathrm{kV}$ and equipped with ChemiSTEM technology. The EDS spectrum image was recorded with a beam current of $1.5 \mathrm{nA}, 0.8 \mathrm{~nm}$ pixel spacing, and a dwell time of $4 \mu \mathrm{spixel}^{-1}$. The elemental maps were calculated from the EDS spectrum image using the In $\mathrm{La}_{1}, \mathrm{Ga} \mathrm{Ka}_{1}$, and $\mathrm{Ga} \mathrm{Ka}_{1}$ lines. Additional element maps can be found in Supplementary Note 3.

Photoluminescence spectroscopy. PL spectroscopy was performed under optical pumping with a ps-pulsed supercontinuum laser (NKT Extreme Red, repetition rate of $78 \mathrm{MHz}$ ). Three emission lines of the ps-pulsed supercontinuum laser at 710,720 , and $750 \mathrm{~nm}$ are used to optically excite the nanostructures. The pump light was focused onto the devices from the top using a $10 \times$ objective. With the same objective, the optical response is collected and free-space coupled to a spectrometer (Princeton SP-2500i) with a cryogenically cooled InGaAs CCD camera (Princeton Instruments, PyLoN-IR, cutoff wavelength of $\sim 1600 \mathrm{~nm}$ ). The setup enables cooling of the samples down to about $10 \mathrm{~K}$, however, all measurements in the main text are performed at room temperature. Low temperature PL measurements can be found in Supplementary Note 8. A typical device-under test (DUT) is depicted in Supplementary Fig. 2c.

Electroluminescence spectroscopy. EL measurements are performed in the same setup as PL spectroscopy. To eliminate any thermal damage, a pulse generator is used and a rectangular voltage function is applied with a repetition rate of $10 \mathrm{kHz}$ and a duty cycle of $50 \%$. The EL is again collected from the top using a $10 \times$ objective and coupled into the spectrometer. Integration times of 10-50 $\mathrm{s}$ are used to obtain a high signal-to-noise ratio.

Spectral response. The spectral response of the devices is measured using the setup described in the previous sections. A ps-pulsed supercontinuum laser excites the devices from the top (10× objective) and the electrical response is measured using a semiconductor device parameter analyzer (Keysight, B1500A). The excitation wavelength is swept from 900 to $1800 \mathrm{~nm}$. The electrical signal is maximized by adjusting the focus at every measured wavelength.

Simulations. First, 2D Finite-Difference-Time-Domain (FDTD) optical simulations using "Sentaurus EMW Solver" are performed to produce the optical generation rate profile in the entire $p-i-n$ structure. A monochromatic light source with constant optical power of $6 \mathrm{dBm}$ is placed at $2 \mu \mathrm{m}$ above the device under an incident angle of $10^{\circ}$ to normal to mimic the free-space excitation. This optical generation profile is then taken as input in the electrical transport simulation using "Sentaurus Device" to calculate the device current at given excitation wavelengths, however we assume an average incident power rather than a pulsed source. By repeating the simulation flow for different excitation wavelengths (from 1200 to $1700 \mathrm{~nm}$, with a step of $50 \mathrm{~nm}$ ), the spectral-response curve can be obtained. The simulated $p-i-n$ device has a height of $60 \mathrm{~nm}$ and a total length of $1.3 \mu \mathrm{m}$. Metal contacts and SOI substrate are included in the FDTD simulations, with $4 \mu \mathrm{m}$ thick air attached to the device on top and at the sides. A constant indium composition of $55 \%$ along the $p-i-n$ structure is assumed for simplicity. The doping concentration is set to the experimentally measured value (see Supplementary Note 2). Shockley-Read-Hall lifetime for all carriers is set to $1 \mathrm{~ns}$ as is well-known for III-V materials, and electron and hole mobility in InGaAs is assumed to be 1100 and $250 \mathrm{~cm}^{2} \mathrm{~V}^{-1} \mathrm{~s}^{-1}$, respectively.

High-speed measurements. Responsivity and high-speed measurements were performed in a second optical setup under illumination with an optical fiber (NA 0.14 ) and a cw laser at $1346 \mathrm{~nm}$. The optical fiber is coupled free-space and impinges under a $10^{\circ}$ angle from the normal. To ensure the highest overlap of the impinging light and the $p-i-n$ structure, the position of the optical fiber was optimized (distance to surface $\sim 2 \mu \mathrm{m}$ ). Measurements are performed on cross-like structures since the contact design fitted well with the RF probes setup (see Supplementary Note 4). The voltage is applied between contacts 1 and 4 as indicated in Supplementary Fig. 2b. A typical DUT is depicted in Supplementary Fig. 2d. Using a vector network analyzer (VNA) the scattering parameter $S_{21}$ of our devices is measured from $100 \mathrm{MHz}$ to $30 \mathrm{GHz}$.

Reporting summary. Further information on research design is available in the Nature Research Reporting Summary linked to this article.

\section{Data availability}

The authors declare that the data supporting the findings of this study are available with the paper and its Supplementary Information files. The data that support the findings of this study are available from the corresponding author upon reasonable request.

Received: 18 November 2019; Accepted: 7 August 2020;

Published online: 11 September 2020

\section{References}

1. Cisco. Cisco visual networking index: forecast and tends, 2017-2022 [White Paper]. Cisco Forecast Methodol. https://www.cisco.com/c/en/us/solutions/ collateral/executive-perspectives/annual-internet-report/white-paper-c11741490.html (2019).

2. Meindl, J. D. Beyond Moore's Law: the interconnect era. Comput. Sci. Eng. 5, 20-24 (2003).

3. Delforge, P. \& Whitney, J. Data center efficiency assessment. Nat. Resour. Def. Counc. Issue Paper, 14-08-a (2014).

4. Miller, D. A. B. Attojoule optoelectronics for low-energy information processing and communications. J. Light. Technol. 35, 346-396 (2017).

5. Atabaki, A. H. et al. Integrating photonics with silicon nanoelectronics for the next generation of systems on a chip. Nature 556, 349-354 (2018).

6. Sun, C. et al. Single-chip microprocessor that communicates directly using light. Nature 528, 534-538 (2015).

7. Wang, Z. et al. Novel light source integration approaches for silicon photonics. Laser Photon. Rev. 11, 1700063 (2017).

8. Chen, H. et al. 100-Gbps RZ data reception in 67-GHz Si-contacted germanium waveguide p-i-n photodetectors. J. Light. Technol. 35, 722-726 (2017).

9. Vivien, L. et al. Zero-bias $40 \mathrm{Gbit} / \mathrm{s}$ germanium waveguide photodetector on silicon. Opt. Express 20, 1096-1101 (2012).

10. Chen, R. et al. Nanolasers grown on silicon. Nat. Photon. 5, 170-175 (2011).

11. Chen, S. et al. Electrically pumped continuous-wave III-V quantum dot lasers on silicon. Nat. Photon. 10, 307-311 (2016).

12. Mauthe, S. et al. InP-on-Si optically pumped microdisk lasers via monolithic growth and wafer bonding. IEEE J. Sel. Top. Quantum Electron. 25, 1-7 (2019).

13. Shi, B. et al. $1.55 \mu \mathrm{m}$ room-temperature lasing from subwavelength quantumdot microdisks directly grown on (001) Si. Appl. Phys. Lett. 110, 121109 (2017).

14. Li, N. et al. Ultra-low-power sub-photon-voltage high-efficiency light-emitting diodes. Nat. Photon. 13, 588-592 (2019).

15. Chen, R. et al. Nanophotonic integrated circuits from nanoresonators grown on silicon. Nat. Commun. 5, 4325 (2014).

16. Kim, H., Farrell, A. C., Senanayake, P., Lee, W.-J. J. \& Huffaker, D. L. Monolithically integrated InGaAs nanowires on 3D structured silicon-oninsulator as a new platform for full optical links. Nano Lett. 16, 1833-1839 (2016).

17. Miao, J. et al. Single InAs nanowire room-temperature near-infrared photodetectors. ACS Nano 8, 3628-3635 (2014).

18. Farrell, A. C., Senanayake, P., Meng, X., Hsieh, N. Y. \& Huffaker, D. L. Diode characteristics approaching bulk limits in GaAs nanowire array photodetectors. Nano Lett. 17, 2420-2425 (2017).

19. Yan, X., Li, B., Wu, Y., Zhang, X. \& Ren, X. A single crystalline InP nanowire photodetector. Appl. Phys. Lett. 109, 053109 (2016)

20. Tan, H. et al. Single-crystalline InGaAs nanowires for room-temperature highperformance near-infrared photodetectors. Nano-Micro Lett. 8, 29-35 (2016).

21. Dai, X. et al. GaAs/AlGaAs nanowire photodetector. Nano Lett. 14, 2688-2693 (2014).

22. Chiba, K., Yoshida, A., Tomioka, K. \& Motohisa, J. Vertical InGaAs nanowire array photodiodes on Si. ACS Photon. 6, 260-264 (2019).

23. Brenneis, A. et al. Photocurrents in a single InAs nanowire/silicon heterojunction. ACS Nano 9, 9849-9858 (2015).

24. Li, D. et al. Ultra-fast photodetectors based on high-mobility indium gallium antimonide nanowires. Nat. Commun. 10, 1664 (2019).

25. Schmid, H. et al. Template-assisted selective epitaxy of III-V nanoscale devices for co-planar heterogeneous integration with Si. Appl. Phys. Lett. 106, 233101 (2015). 
26. Cutaia, D. et al. Complementary III-V heterojunction lateral NW tunnel FET technology on Si. In 2016 IEEE Symposium on VLSI Technology, Honolulu, HI, 2016, pp. 1-2 (IEEE, 2016).

27. Borg, M. et al. Vertical III-V nanowire device integration on Si(100). Nano Lett. 14, 1914-1920 (2014)

28. Borg, M. et al. High-mobility GaSb nanostructures cointegrated with InAs on Si. ACS Nano 11, 2554-2560 (2017).

29. Bologna, N. et al. Dopant-induced modifications of Ga $\mathrm{xIn}(1-\mathrm{x}) \mathrm{P}$ nanowirebased p-n junctions monolithically integrated on Si(111). ACS Appl. Mater. Interfaces 10, 32588-32596 (2018).

30. Borg, M. et al. Facet-selective group-III incorporation in InGaAs template assisted selective epitaxy. Nanotechnology 30, 084004 (2019).

31. Synopsys, Inc., Synopsys Sentaurus Device (S-Device) \& Electromagnetic Wave (EMW) Solver, Version O-2018.06 (2018).

32. Baumgartner, Y. et al. Novel CMOS-compatible ultralow capacitance hybrid III-V/Si photodetectors tested up to $32 \mathrm{Gbps}$ NRZ. In Optical Fiber Communication Conference. OSA Technical Digest paper Th3B.3 (Optical Society of America, 2019).

33. Lee, Y.-L., Huang, C.-C., Ho, C.-L. \& Wu, M.-C. Planar InGaAs p-i-n photodiodes with transparent-conducting-based antireflection and doublepath reflector. IEEE Electron. Device Lett. 34, 1406-1408 (2013).

34. Nadtochiy, A. M. et al. Time-resolved photoluminescence of InGaAs nanostructures different in quantum dimensionality. Semiconductors 53, 1489-1495 (2019).

35. Li, Y. et al. Selective area growth of InGaAs/InP quantum well nanowires on SOI substrate. ECS Trans. 86, 115-121 (2018).

\section{Acknowledgements}

The authors acknowledge financial support from Horizon 2020 European Research Council project PLASMIC grant no. 678567 and Horizon 2020 project DIMENSION grant no. 688003. We acknowledge E. Salis, H. Schmid, and J. Leuthold for technical discussions and assistance. We acknowledge S. Hönl for graphic support.

\section{Author contributions}

S.M. designed and fabricated the diodes. S.M. grew the $p-i-n$ InGaAs heterostructure. S.M. and M.S. prepared focused ion beam lamellas for material analysis. M.S. and M.D.R. performed detailed material characterization. S.M., Y.B., and L.C. conducted the electrooptical characterization. Q.D. and A.S. performed electro-optical simulation. S.M. wrote the article with contribution from all authors. K.E.M. and L.C. led the project.

\section{Competing interests}

The authors declare no competing interests.

\section{Additional information}

Supplementary information is available for this paper at https://doi.org/10.1038/s41467 020-18374-z.

Correspondence and requests for materials should be addressed to K.E.M.

Peer review information Nature Communications thanks Yannick De Koninck and the other, anonymous, reviewer(s) for their contribution to the peer review of this work.

Reprints and permission information is available at http://www.nature.com/reprints

Publisher's note Springer Nature remains neutral with regard to jurisdictional claims in published maps and institutional affiliations.

cc (i) Open Access This article is licensed under a Creative Commons Attribution 4.0 International License, which permits use, sharing, adaptation, distribution and reproduction in any medium or format, as long as you give appropriate credit to the original author(s) and the source, provide a link to the Creative Commons license, and indicate if changes were made. The images or other third party material in this article are included in the article's Creative Commons license, unless indicated otherwise in a credit line to the material. If material is not included in the article's Creative Commons license and your intended use is not permitted by statutory regulation or exceeds the permitted use, you will need to obtain permission directly from the copyright holder. To view a copy of this license, visit http://creativecommons.org/ licenses/by/4.0/.

(C) The Author(s) 2020 